\title{
符号熵驱动的零件机加工艺过程的多维度相似性度量方法
}

\author{
李春磊 1,2$)$ ，王肖烨 1,2 ，李亮 1,2$)$ \\ 1) (宝鸡文理学院机械工程学院 宝鸡 721016) \\ 2) (陕西省机器人关键零部件先进制造与评估省市共建重点实验室 宝鸡 721016) \\ (6021c1-6021c1@163.com)
}

\begin{abstract}
摘 要: 机加工艺过程从表象看是一道道工序加工方法组成的序列, 但从内里看其实是工件从初始毛坏模型到最终 成品模型的几何结构演变过程，单纯从工序加工序列或三维几何结构的角度出发进行相似性检索会降低检索到工艺 的有效性. 针对以上问题，提出一种符号熵驱动的零件机加工艺过程的多维度相似性度量方法. 首先建立基于符号 熵的序列相似性度量方法，直接对加工方法序列进行相似性计算；然后建立与加工方法序列对应的几何演变序列， 并在符号熵方法的支持下实现对几何演变过程的相似性度量；最后将加工方法序列的相似性计算结果及对应几何演 变过程的相似性计算结果进行融合，实现对机加工艺过程相似性的多维度和精准评价. 实例结果表明，所提方法在 度量不同类型机械零件的工艺相似性时，评价结果更贴近实际制造工艺过程和工艺人员的经验认知，证明方法是可 行的.
\end{abstract}

关键词: 符号熵; 机加工艺过程; 几何演变序列; 多维相似性度量

中图法分类号: TP391.41 DOI: 10.3724/SP.J.1089.2021.18735

\section{Multidimensional Similarity Measurement Method of Machining Processes Driven by Sign Entropy}

\author{
Li Chunlei ${ }^{1,2)}$, Wang Xiaoye ${ }^{1,2)}$, and Li Liang ${ }^{1,2)}$ \\ 1) (School of Mechanical Engineering, Baoji University of Arts and Sciences, Baoji 721016) \\ 2) (Shaanxi Key Laboratory of Advanced Manufacturing and Evaluation of Robot Key Components, Baoji 721016)
}

\begin{abstract}
From the outside, the machining process seems a process sequence formed by processing operations one by one. But from the inside, it is a geometry variation process from the initial blank model to final CAD model. It is clear that individually measuring the similarity of processing operation sequences or geometry variation sequences will reduce the effectiveness of machining process similarity retrieval. To solve this problem, a multidimensional similarity measurement method of machining processes driven by sign entropy is presented. First, a similarity measurement way of sequence based on sign entropy is proposed, which can directly realize the similarity measurement of processing operation sequences. Then, the geometry variation sequence corresponding to processing operation sequence is established, and further achieved the goal of measuring the similarity of geometry variation sequences in the support of the sign entropy way. Finally, make the above two similarity measurement results are fused together, and thus evaluating the similarity of machining processes is evaluated multidimensionally and accurately. Case studies show that the evaluation result by using proposed method to measure the similarity of different kinds of parts is closer to actual manufacturing process and cog-
\end{abstract}

收稿日期: 2020-10-23; 修回日期: 2021-06-05. 基金项目: 陕西省教育厅自然科学类专项科研计划(21JK0490); 陕西省自然科学 基础研究计划(2019JQ-896). 李春否(1987-), 男, 博士, 讲师, 主要研究方向为智能制造、知识工程; 王肖烨(1975一), 女, 博士, 副教 授, 硕士生导师, 主要研究方向为工业设计; 李亮(1979-), 男, 博士, 副教授, 硕士生导师, 主要研究方向为机器人整机及关键零部 件检测. 
nitive experience, which proves that the proposed method is feasible.

Key words: sign entropy; machining process; geometry variation sequence; multidimensional similarity measurement

制造业作为国民经济的支柱领域之一，对其 进行转型升级和提质增效具有非常迫切的现实意 义. 而随着人工智能、物联网、大数据以及新一代 通信技术等新兴科技的不断涌现及广泛应用，推 动基于智能制造理念的制造模式创新已成为学术 界和产业界的共识. 制造过程的智能特性主要表 现在系统能够基于已有的案例数据、知识、经验和 规律对制造工艺过程进行自组织和安排. 因此，对 已有的工艺案例进行有效的挖掘分析，提取其中 的相似工艺案例，既是找寻同类型工艺案例中隐 含的共性知识和规律的前提，更是后续面对新的 类似加工对象时，通过借鉴已有案例快速推理和 智能捊化生成新的加工案例的重要依据.

在评价工艺相似性时, 最直接的思路就是对 工序序列进行相似性度量. 例如, Liu 等 ${ }^{[1]}$ 将机加 $^{-}$ 工艺过程简化成为工艺路线，并基于经典的距离 计算方法构建了工艺路线的相似性度量公式. 张 辉等 ${ }^{[2]}$ 基于提取到的最长公共子序列，提出了序列 的多级相似性综合度量模型，可以实现对加工方 法序列的相似性计算. 周丹晨 ${ }^{[3]}$ 将工序序列抽象成 基因序列，然后利用生物信息学中的基因比对技 术获取 2 条待度量序列的最佳比对，最后基于最佳 比对构建了工序路线的相似性计算模型. 范午阳 ${ }^{[4]}$ 针对同类型相似零件的工序序列长度未必相同的 问题，引人了用于集合相似性度量的谷本系数，可 针对长度不同的工序序列直接进行相似性判断, 从而更有效地解决不同型号零件间的工艺路线相 似性判定问题. 曹勇 ${ }^{[5]}$ 在文献[2]方法的基础上，提 出了一种基于多级最长公共子序列的工艺路线相 似性度量方法. 以上方法的总体思路都是将工艺 过程看做由工序加工方法组成的序列，通过构建 序列的相似性计算模型来比较不同工艺过程间的 相似性. 但事实上，同样的工序方法可能产生不同 的加工成果，而且随着工艺过程的工件几何结构 的逐渐演化完善，也无法用一个简单序列进行表 示，这种单一的检索条件已经难以满足日益复杂 和多样化的工艺相似性检索需求.

除上述方法之外, 还有学者从工件模型的几 何结构角度出发对工艺过程的相似性度量进行研
究. 这类方法是在“相似几何结构具有相似工艺” 的思想指导下, 先利用图论和降维等技术 ${ }^{[6-9]}$ 将三 维计算机辅助设计 (computer aided design, CAD) 模 型转化成可供度量的数学表达形式, 再利用图同 构 ${ }^{[10-11]}$ 和特征向量 ${ }^{[12-13]}$ 检索出与模型对象具有最 大几何结构相似性的工艺案例. 但这类方法的一 个明显不足是未考虑同样的几何结构可能是通过 不同加工手段实现的，而且静态的几何结构也无 法对动态的工艺过程进行精准刻画. 因此, 这也不 是一种完备的工艺过程相似性度量方法. 近年来, 已经有学者注意到伴随加工进程的工件结构的几 何演变才是衡量工艺过程相似性的直接依据, 并 给出了工序模型几何演变过程的建模方法和应用 范例 ${ }^{[14-16]}$ ，但依然欠缺可供精准表达和快速计算 的成熟方式，而且检索维度的相对单一也限制了 这类方法的适用性.

事实上，机加工艺过程从表象看是一道道工 序加工方法组成的序列, 但从内里看其实是工件 从初始毛坏模型到最终成品模型的几何结构演变 过程, 而且几何结构的改变与工序加工操作直接 相关. 即机加工艺过程有着工序加工序列和几何 演变序列 2 层动态变化属性, 将二者进行融合考虑 有利于形成检索条件更加丰富、评判标准更加全 面、度量过程更加精确的工艺过程性相似性计算模 式. 鉴于此, 本文提出了一种符号熵驱动的工艺过 程多维度相似性度量方法. 首先建立了基于符号 熵的序列相似性度量模型, 实现对加工方法序列 的相似性计算. 因为符号熵的产生综合考虑了 2 条 原序列中共有工序符号的出现频次和分布密度等 信息，所以使相似性评判结果能够兼顾序列的整 体结构相似性和局部共有工序的相似性, 评判结 果更加可靠. 进一步，建立了与加工方法序列对应 的几何演变序列, 并将几何结构改变的基础单元 降维转化成唯一对应的字符串表达形式, 为构建 基于符号熵的方法从底层突破几何结构度量的问 题, 并辅以集合相似性度量、最佳匹配序列比对等 方式, 实现几何演变过程的相似性度量. 最后, 融 合加工方法序列的相似性计算结果及对应几何演 变过程的相似性计算结果, 实现对机加工艺过程 
的多维度和精准评价.

\section{1 基于符号熵加工方法序列的相似性度量}

现代复杂产品的机械加工过程往往涉及多道 工序，而且这些工序一般是在不同工位(设备)上完 成的. 产品的加工方法序列反映了产品加工工序 的先后安排次序, 内含了机加工艺过程需要调用 的制造资源类型及其调用次序，以及工件在多工 位(设备)间的流转路径. 显然, 2 个零件的加工方 法序列越相似，说明其工艺过程调用的制造资源 类型、数量、次序以及工件在加工现场中的传输路 径也越相似, 工艺方案的一致性也越高.

本文使用基于符号熵的序列相似性度量方法 评价加工方法序列间的相似性. 首先, 从待比较的 2 个加工方法序列中，提取最长公共子符号序列; 然后，将每种加工方法抽象成一种符号，计算最长 公共子序列中每个位置符号的香农熵，并利用所 有位置的熵之差组成子序列的相似性评判结果. 因为符号熵的计算结果与该符号所处位置及出现 次数有关, 所以最终得到的子序列相似性计算结 果可以有效地反映序列的全局与局部信息，增强 了计算结果的可靠性. 最后, 综合考虑最长公共子 序列的相似性评价结果以及待比较的 2 条原序列 长度, 得到一个比较准确且可靠并能够兼顾序列 局部信息、整体信息以及长度信息的加工方法序列 的相似性计算结果.

\section{1 最长公共子序列的获取}

最长公共子序列的获取是一个在 2 条待比较 序列中查找最长子序列的问题. 最长公共子序列 的定义如下: 若某个序列 $S=\left\{s_{1}, s_{2}, \cdots, s_{r}\right\}$ 同时是 2 条待比较序列 $M=\left\{m_{1}, m_{2}, \cdots, m_{u}\right\}$ 和 $N=\left\{n_{1}\right.$, $\left.n_{2}, \cdots, n_{v}\right\}$ 的子序列, 即满足

$$
\begin{cases}s_{x}=m_{y_{x}}=n_{z_{x}}, & 1 \leqslant x \leqslant r \\ 1 \leqslant y_{x} \leqslant u, & x=1,2, \cdots, r \\ 1 \leqslant z_{x} \leqslant v, & x=1,2, \cdots, r\end{cases}
$$

当且仅当序列 $S$ 的长度 $r$ 取最大值时，称 $S$ 为 $M$ 和 $N$ 的最长公共子序列.

最长公共子序列的获取是生物信息学应用的 基础，也是一个比较经典的计算机动态规划求解 问题，文献[17-18]和一些工具(如 Diff 工具)针对此 问题已经形成了比较成熟和通用的解决方法，这 里不再赘述.

\section{2 符号熵的计算}

本文采用香农熵对加工方法序列中各个位置 工序的符号熵进行计算. 对于序列 $M$, 同一工序符 号可能在 $M$ 中出现多次, 则某工序符号在 $M$ 中第 $i$ 次出现的位置信息及其出现密度描述式为

$$
\alpha_{i}=\frac{1}{p_{i}-p_{i-1}}, \quad 1 \leqslant i \leqslant k
$$

其中， $p_{i}$ 为该工序符号在序列中第 $i$ 次出现时的位 置; $k$ 为该工序符号在序列中的出现次数. 显然, $p_{0}$ 可以看做该工序符号还未出现时的位置, 定义 $p_{0}=0$. 由式(2)可知, 当工序符号在序列中出现的 位置越靠后，且与前一次出现的间隔越大时， $\alpha_{i}$ 值越小; 反之, $\alpha_{i}$ 值越大. 因此, $\alpha_{i}$ 可以对工序符 号第 $i$ 次出现的位置及该位置与同符号上一次出现 的密集情况加以描述.

为了描述 $\alpha_{i}$ 的有序性，可以构建离散概率分 布函数

$$
Q_{i}=\sum_{j=1}^{i} \alpha_{j} / \sum_{j=1}^{k} \alpha_{j}, \quad 1 \leqslant i, j \leqslant k
$$

进一步, 可以对序列中各个位置上的工序符号计 算香农熵, 如

$$
H\left(P_{t}\right)=-Q_{i} 1 \mathrm{~b}\left(Q_{i}\right), \quad 1 \leqslant i \leqslant k, 1 \leqslant t \leqslant r
$$

其中， $P_{t}$ 为序列 $M$ 中第 $t$ 个工序符号的位置; $H\left(P_{t}\right)$ 为 $P_{t}$ 位置工序的符号熵; $P_{t}$ 位置工序在 $M$ 中是第 $i$ 次出现; $Q_{i}$ 为其分布概率.

\section{3 基于符号熵的序列相似性计算}

对于任意 2 条待比较序列 $M=\left\{m_{1}, m_{2}, \cdots, m_{u}\right\}$ 和 $N=\left\{n_{1}, n_{2}, \cdots, n_{v}\right\}$, 可以提取它们的最长公共子 序列 $S=\left\{s_{1}, s_{2}, \cdots, s_{r}\right\}$. 若 $S$ 中某工序符号 $s_{x}$ 在 $M$ 中的位置为 $P_{y_{x}}$, 在 $N$ 中的位置为 $P_{z_{x}}$, 则 2 条序列 的相似性计算公式为

$$
s_{\mathrm{im}}(M, N)=1-\frac{1}{r} \sum_{x=1}^{r}\left|H\left(P_{y_{x}}\right)-H\left(P_{z_{x}}\right)\right|
$$

其中, $H\left(P_{y_{x}}\right)$ 和 $H\left(P_{z_{x}}\right)$ 分别为 $s_{x}$ 在 $M$ 和 $N$ 中的香 农熵计算结果. 可以看出, 2 条加工方法序列的相 似性计算结果由最长公共子序列中所有符号的香 农熵之差组成. 显然, 如果最长公共子序列在 2 条 原序列中出现的位置及同符号出现的间隔等信息 一致，则计算得到的相似性就越高. 而符号熵又反 映了原序列的整体与局部结构信息, 避免了依靠 子序列度量缺乏考虑整体结构信息的问题，提高 了相似性计算的准确性.

现实中, 如果 2 条待比较序列的长度相差较 
大，即使最长公共子序列在原序列中出现的位置 及频次信息较为接近，也无法依此认定 2 条序列的 相似性较高. 例如, 字符序列 aabedf 和 aabe, 最长 公共子序列为 aabe 和 aabe, 在 2 条原序列中出现 的位置及频次间隔一致，此时不能简单认定 2 条序 列完全相似. 因此, 还需要考虑因为序列长度不一 致带来的影响, 引入能够反映长度不一致的修正 系数，构建出相似性最终计算公式

$$
S_{\text {im }}(M, N)=\frac{r}{\max (u, v)} s_{\text {im }}(M, N)
$$

下面通过一个简单例子说明如何利用符号熵 计算加工方法序列的相似性. 假设存在 2 条加工方 法序列: $M$ (铣削 $\rightarrow$ 磨削 $\rightarrow$ 镗削 $\rightarrow$ 车削 $\rightarrow$ 钻削 $\rightarrow$ 镗 削)和 $N$ (铣削 $\rightarrow$ 磨削 $\rightarrow$ 铣削 $\rightarrow$ 镗削 $\rightarrow$ 钻削). 可以提 取二者的最长公共子序列 $S$ (铣削 $\rightarrow$ 磨削 $\rightarrow$ 镗削 $\rightarrow$ 钻削), 然后利用式(2) 式(4)计算 $S$ 中每个加工元素 分别在 $M$ 和 $N$ 中的符号熵, 计算结果如表 1 所示.

表 1 符号熵计算示例

\begin{tabular}{ccc}
\hline$x$ & $H\left(P_{y_{x}}\right)$ & $H\left(P_{z_{x}}\right)$ \\
\hline 1 & 0 & 0.39 \\
2 & 0 & 0 \\
3 & 0.5 & 0 \\
4 & 0 & 0 \\
\hline
\end{tabular}

将表 1 中数据代人式(5)(6), 可以得到加工序 列相似性度量的最终结果为

$$
S_{\text {im }}(M, N)=\frac{4}{6}\left[1-\frac{1}{4}(|0-0.39|+|0.5-0|)\right] \approx 0.5183 .
$$

\section{2 几何演变驱动下的机加工艺过程的相似 性度量}

从表象看，工件的加工过程是在不同机加工 位间的流转; 但从本质看, 每道工位上的工序加工 都会导致工件结构出现一定程度的改变, 最终体 现为从毛坏模型到最终成品模型的一个几何演变 过程. 因此，几何结构的改变是机加工序执行结果 的最直接体现，对伴随加工进程的几何结构演变 信息进行相似性评判，可以为相似工艺的检索重 用提供更贴近设计本质的依据.

\section{1 匹配机加工序的几何变更的提取和表示}

伴随着工艺过程的持续，工件会从毛坏逐渐 过渡到最终成品, 而每道机加工序操作都是导致 工件发生几何结构改变的直接原因. 对匹配机加
工序的几何结构改变进行提取和表示, 是构造工 艺过程几何演变模型的前提和基础, 也是从几何 演变角度衡量工艺相似性的最基础的评价单元.

现代复杂机械零部件的机加工艺过程往往包 含多道工序，其中任意一道工序造成的几何变更 可以表示为

$$
G_{\mathrm{V}_{-} i}=\sum_{j=1}^{n_{\mathrm{GV}}^{i}} g_{i}^{j}=I_{\mathrm{PM}}^{i-1}-I_{\mathrm{PM}}^{i}
$$

其中, $G_{\mathrm{V}_{-} i}$ 为匹配工艺过程第 $i$ 道机加工序的几何 变更; $g_{i}^{j}$ 为在执行第 $i$ 道工序的过程中, 第 $j$ 次走 刀的扫略体积与工件实体的布尔交, 即加工切除 掉的三维几何结构模型; $I_{\mathrm{PM}}^{i}$ 为工件经历第 $i$ 道工 序加工后呈现出的三维几何结构形态, 如果将最 终成品模型或 CAD 设计模型看做全部工序都完成 后的几何结构形态, 那么 $I_{\mathrm{PM}}^{i}$ 可看做中间过程模 型， $I_{\mathrm{PM}}^{0}$ 则体现为初始毛坏模型的概念. 由式(7)可 知, $I_{\mathrm{PM}}$ 的生成对构建 $G_{\mathrm{V}}$ 至关重要. 目前生成 $I_{\mathrm{PM}}$ 的方式主要有 2 种: 利用基于模型定义的技术 构建 ${ }^{[19-21]}$; 利用工序间的逻辑关系并配合计算机 辅助手段 ${ }^{[22-24]}$ 快速生成.

由几何变更的定义可知，匹配机加工序的几 何变更可看做对前后道工序执行后生成的中间过 程模型进行布尔差操作. 以某阶梯轴零件为例, 原 始毛坏模型为棒材，执行第 1 道粗车工序后形成的 几何变更可用图 1 表示.

根据本文对几何变更的定义, 其可以看做因 执行工序加工走刀而切除掉的三维几何结构模型 的集合, 所以对几何变更的数学表示问题转化为 对三维几何结构模型的集合表示. 应用图论中的 属性邻接图表示三维几何模型, 是当前对三维结 构进行降维数学表达的一种最主要手段. 本文利 用属性邻接图对三维几何结构模型进行表示, 定 义几何变更中三维几何结构模型的属性邻接图为 $A_{\mathrm{AG}}=\left(V^{\prime}, E^{\prime}, V_{\mathrm{AS}}, E_{\mathrm{A}}\right)$. 其中, $V^{\prime}$ 为 $A_{\mathrm{AG}}$ 的顶点集 合, 集合中的顶点 $v^{\prime}$ 与用 $A_{\mathrm{AG}}$ 表达的三维模型表 面间存在一一映射关系; $E^{\prime}$ 为 $A_{\mathrm{AG}}$ 的边集合, 集 合中的边与用 $A_{\mathrm{AG}}$ 表达的三维模型棱边间存在一 一映射关系; $V_{\mathrm{AS}}$ 是用 $A_{\mathrm{AG}}$ 表达的三维模型表面的 属性集，主要包括面的连接度、类型和面积等信息; $E_{\mathrm{A}}$ 是用 $A_{\mathrm{AG}}$ 表达的三维模型棱边的类型属性. 三 维模型的棱边是以 2 个面的邻接边形式存在. 如果 2 个面间不存在邻接关系, 则 $E_{\mathrm{A}}$ 类型为空, 用空 


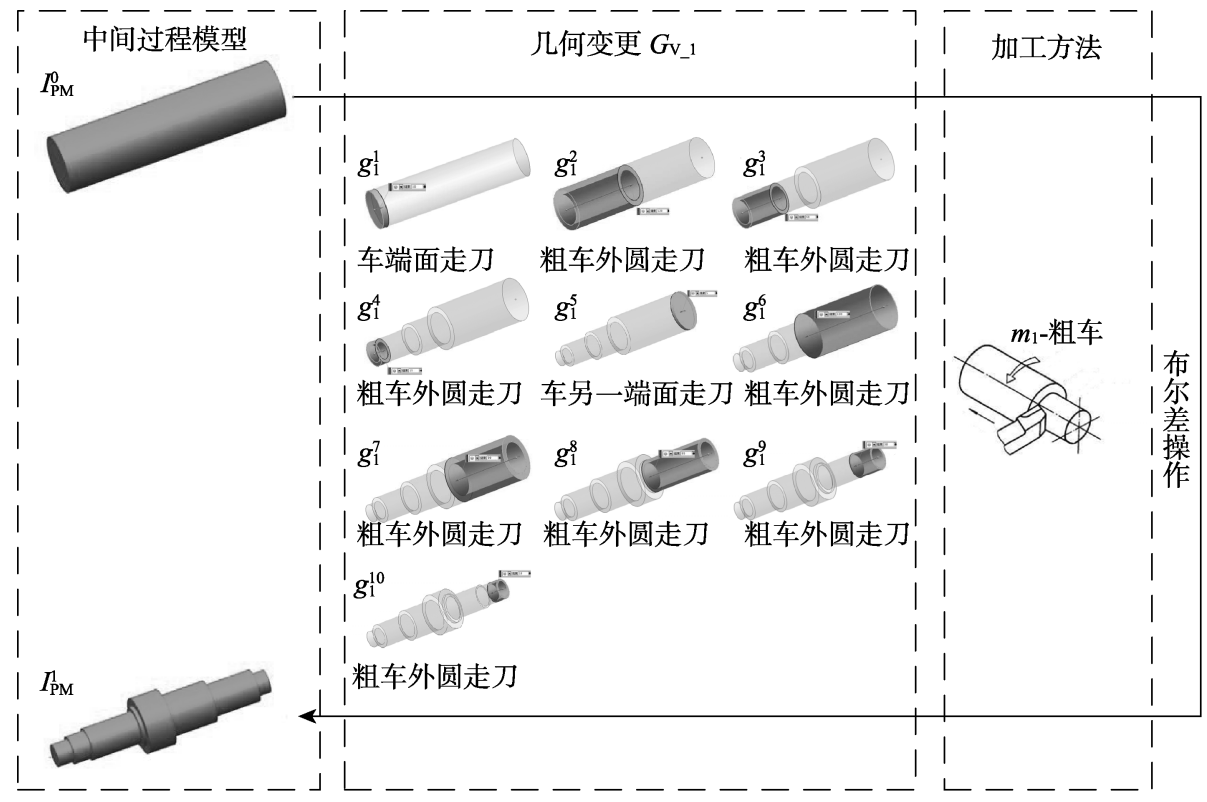

图 1 几何变更的提取示意图

集符号 $\varnothing$ 表示; 如果 2 个面间存在邻接关系，当邻 接边为直线时, 取 $E_{\mathrm{A}}=0$, 当邻接边为平面曲线时, 取 $E_{\mathrm{A}}=1$, 当邻接边为空间曲线时, 取 $E_{\mathrm{A}}=2$. 以图 1 中的 $G_{\mathrm{V}} 1$ 为例, 粗车工序总共包含 10 次走刀, 共 切除掉 10 个三维几何结构模型，它们可以用图 2 所示对应的属性邻接图表示.

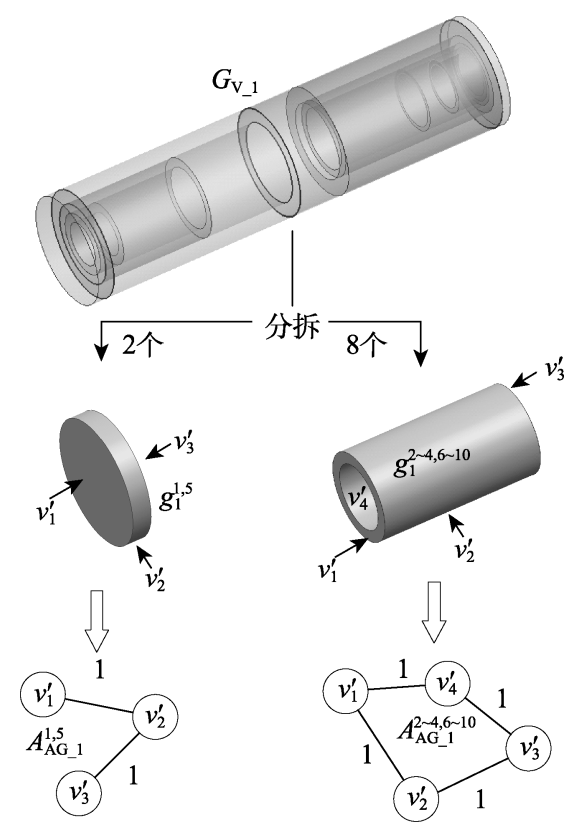

图 2 几何变更的图表示

图表示方法虽然能够对几何变更的三维几何 结构信息进行准确描述, 但并不利于存储和计算 分析, 因此, 有必要对其转化. 本文采用字符串转 化方法 ${ }^{[25]}$ 对 $A_{\mathrm{AG}}$ 进行进一步的降维表达.
进行字符串转化的思路是先按照一定策略对 $A_{\mathrm{AG}}$ 中的顶点进行排序. 对于属性邻接图中的 2 个 顶点, 可以先评价对应面的连接度, 如果一个面的 连接度大于另一个面的连接度, 则具有较大连接 度的面排在前面; 如果 2 个顶点对应面的连接度相 同，则按照面的类型进行排序，一般遵循“平面先 于圆柱面，圆柱面先于圆雉面，圆雉面先于其他类 型的曲面”的排序原则; 如果 2 个顶点对应面的连 接度和面的类型均相同, 则根据面积大小进行排 序, 顶点对应面的面积大的排前面. 属性邻接图的 顶点经排序后会形成一个具有前后位置关系的顶 点序列，如果其中每个顶点用对应面的类型字符 表示，并且在所有前后 2 个顶点间插人它们对应的 邻接边属性值字符, 那么就形成了一个唯一对应 的字符串.

本文定义平面的表示字符为 $P_{l}$, 圆柱面的表 示字符为 $C_{y}$, 圆雉面的表示字符为 $C_{i}$, 螺纹面的 表示字符为 $T_{h}$. 排序后相邻顶点间的属性值字符 可以从原 $A_{\mathrm{AG}}$ 中的 $E_{\mathrm{A}}$ 提取.

以图 2 所示的 $A_{\mathrm{AG}}$ 为例, 按照字符串转化策略 对其进行处理, 形成如图 3 所示的顶点重新排序结 果. 由图 3 可知, $A_{\mathrm{AG} 11}^{1}$ 和 $A_{\mathrm{AG} 1}^{5}$ 可以用唯一确定的 字符串 $C_{y} 1 P_{l} P_{l} \phi C_{y}$ 表示； $A_{\mathrm{AG} \_1}^{2}, A_{\mathrm{AG} \_1}^{3}, A_{\mathrm{AG} \_1}^{4}$, $A_{\mathrm{AG} \_1}^{6}, A_{\mathrm{AG}_{-} 1}^{7}, A_{\mathrm{AG} \_1}^{8}, A_{\mathrm{AG}_{-} 1}^{9}$ 和 $A_{\mathrm{AG} \_1}^{10}$ 可以用唯一确 定的字符串 $P_{l} \phi P_{l} P_{l} 1 C_{y} C_{y} \phi C_{y}$ 表示. 这样, 原本由 10 个 $A_{\mathrm{AG}}$ 构成的 $G_{\mathrm{V} 1}$ 就转化成为由 10 个字符串组 成的集合。 


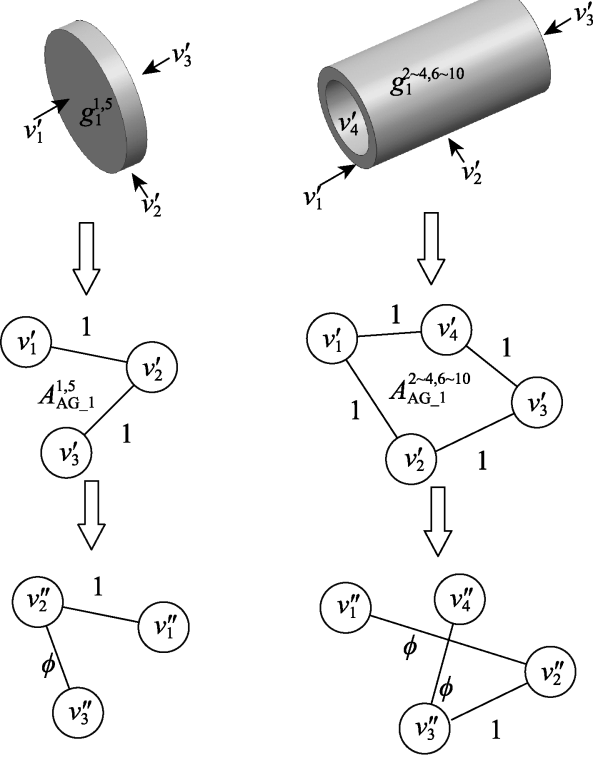

图 3 顶点进行排序后的属性邻接图表示

\section{2 构造与加工方法序列匹配的几何演变序列}

对于某加工方法序列 $M=\left\{m_{1}, m_{2}, \cdots, m_{u}\right\}$, 匹 配中任意一道机加工序 $m_{i}(1 \leqslant i \leqslant u)$ 的几何变更如 果用 $G_{\mathrm{V}_{i}}$ 进行表示. 那么按照加工方法序列中的 工序前后优先级关系，可以构造出与 $M$ 相匹配的 几何演变序列

$$
G_{\mathrm{VS}}=\left\{G_{\mathrm{V}_{-} 1}, G_{\mathrm{V}_{-} 2}, \cdots, G_{\mathrm{V}_{-} u}\right\}
$$

其中， $G_{\mathrm{VS}}$ 表示匹配加工方法序列 $M$ 的几何演变 序列; $G_{\mathrm{V} i}(1 \leqslant i \leqslant u)$ 表示匹配机加工序 $m_{i}$ 的几何 变更，它可以用字符串集合进行表示.

需要注意的是，严格按照工序序列构建对应 的 $G_{\mathrm{VS}}$ 虽然能够对完整地反映加工过程，但从几 何结构形态的改变来看存在冗余信息. 如一些半 精加工、精加工以及磨削等工序，只是为了促使一 些加工尺寸逐步递进满足设计精度要求. 因此, 后 续在评价 $G_{\mathrm{VS}}$ 间的相似性时, 可以忽视这些只为 满足质量精度要求的圥余几何变更，提取构造出 关键几何演变序列 $G_{\mathrm{VS}}^{*}$.

\section{3 几何演变序列的相似性度量}

根据本文建立的几何变更的表示方式和几何 演变序列的构造定义，要想实现对几何演变序列 的相似性评价, 先要实现对几何变更的相似性计 算，而几何变更又是由字符串组成的集合，所以最 初落脚点是要实现对字符串的相似性度量. 按照 这一关系，几何演变的相似性度量应按照“字符串 相似性一几何变更相似性一几何演变序列相似性” 的逻辑关系逐步递进实现.

首先计算字符串的相似性，如果将字符串中
的每个字符都视作符号, 那么字符串就成为一个 符号序列，可以直接套用第 1 节基于符号熵的序列 相似性度量方法，这里不再赘述。

其次，几何变更的本质是一个字符串集合. 常 规的计算 2 个集合相似性的方法是先求 2 个集合的 交集和并集，然后用交集包含的元素个数除以并集 包含的元素个数, 即从 2 个集合包含相同元素个数 的角度进行相似性评判. 但几何变更中的字符串元 素表示的是一次切削走刀去除掉的三维几何结构模 型，而模型几何结构的些许差异不能完全排除工艺 上可能存在相似性, 所以从包含元素完全一致的角 度计算字符串集合的相似性，可能造成工艺搜索的 “漏检”. 基于此，本文改进并优化了字符串集合的 相似性计算方法，对于 2 个待比较的字符串集合 $G_{\mathrm{V}}=\left\{S_{\mathrm{tr} \_1}, \cdots, S_{\mathrm{tr} \_}, \cdots\right\}$ 和 $G_{\mathrm{V}}^{\prime}=\left\{S_{\mathrm{tr}_{-} 1}^{\prime}, \cdots, S_{\mathrm{tr}_{-} j}^{\prime}, \cdots\right\}$, 假设 $G_{\mathrm{V}}$ 中元素数量 $\left|G_{\mathrm{V}}\right|$ 不小于 $G_{\mathrm{V}}^{\prime}$ 中元素数量 $\left|G_{\mathrm{V}}^{\prime}\right|$; 从 $G_{\mathrm{V}}$ 中找出 $\left|G_{\mathrm{V}}^{\prime}\right|$ 个字符串元素, 进一步与 $G_{\mathrm{V}}^{\prime}$ 中的元素实现两两互相匹配, 并且让所有匹配 对间的字符串相似性计算值的和最大，即让 2 个集 合按照彼此元素间的相似性值和进行最佳匹配， 使最终匹配到的相似性和值最大. 最大相似性和 值可以认为是 $G_{\mathrm{V}}$ 和 $G_{\mathrm{V}}^{\prime}$ 共有的 “相同”元素个数, 则可以构建几何变更的相似性计算公式

$$
s_{\mathrm{mgv}}\left(G_{\mathrm{V}}, G_{\mathrm{V}}^{\prime}\right)=\frac{\max \left(\sum s_{\mathrm{mstr}}\left(S_{\mathrm{tr}_{-} i}, S_{\mathrm{tr}_{-} j}^{\prime} j\right)\right)}{\left|G_{\mathrm{V}}\right|+\left|G_{\mathrm{V}}^{\prime}\right|-\max \left(\sum s_{\mathrm{mstr}}\left(S_{\mathrm{tr}_{-} i}, S_{\mathrm{tr}_{-} j}^{\prime} j\right)\right)}
$$

其中, $s_{\mathrm{mgv}}\left(G_{\mathrm{V}}, G_{\mathrm{V}}^{\prime}\right)$ 表示 $G_{\mathrm{V}}$ 和 $G_{\mathrm{V}}^{\prime}$ 的相似性计算 值; $\max \left(\sum s_{\mathrm{mstr}}\left(S_{\mathrm{tr}_{-} i}, S_{\mathrm{tr}_{-} j}^{\prime}\right)\right)$ 表示匹配到的最大相 似性和值结果. 因为 2 个集合按照某一元素对应关 系进行最佳匹配是一个经典的数学问题, 且现有 很多方法都可以完美求解，所以这里不再赘述.

最后，计算几何演变序列的相似性，其实现思 路是先获取 2 条待比较序列的最佳匹配序列, 再计 算最佳匹配序列的相似性; 同时考虑待比较序列 的长度对相似性计算值的影响, 并进行修正, 最终 得到 2 条序列的相似性度量结果.

对于 2 条待比较的几何演变序列 $G_{\mathrm{VS} 1}$ 和 $G_{\mathrm{VS} \_2}$, 可以先以 $G_{\mathrm{VS} \_1}$ 为基础构建关键几何演变 序列 $G_{\mathrm{VS} \_1}^{*}$, 再从 $G_{\mathrm{VS}_{2} 2}$ 中提取能与 $G_{\mathrm{VS} 1}^{*}$ 实现最佳 匹配的序列 $G_{\mathrm{VS} 2}^{\prime}$. $G_{\mathrm{VS} 2}^{\prime}$ 的构造过程如下.

Step1. 将 $G_{\mathrm{VS} \_1}^{*}$ 中的第 1 个几何变更与 $G_{\mathrm{VS}_{2} 2}$ 中的 所有几何变更都进行相似性计算, 取其中对应计算值最 
大的 $G_{\mathrm{VS} 2}$ 中元素作为 $G_{\mathrm{VS} 22}^{\prime}$ 的第 1 个元素.

Step2. 更新 $G_{\mathrm{VS} \_2}$ 序列, 删除 $G_{\mathrm{VS} \_2}$ 中从 $G_{\mathrm{VS} \_2}^{\prime}$ 中 取到的元素及其位置之前的元素, 形成新的 $G_{\mathrm{VS} \_2}$ 序列 并参与后续比较.
Step3. 重复上述过程, 直到 $G_{\mathrm{VS} 1}^{*}$ 中的所有元素都 依次找到了最佳匹配元素, 或者新形成的 $G_{\mathrm{VS} \_2}$ 序列成 为了空序列为止.

最佳匹配序列的获取过程可以用图 4 进行描述.

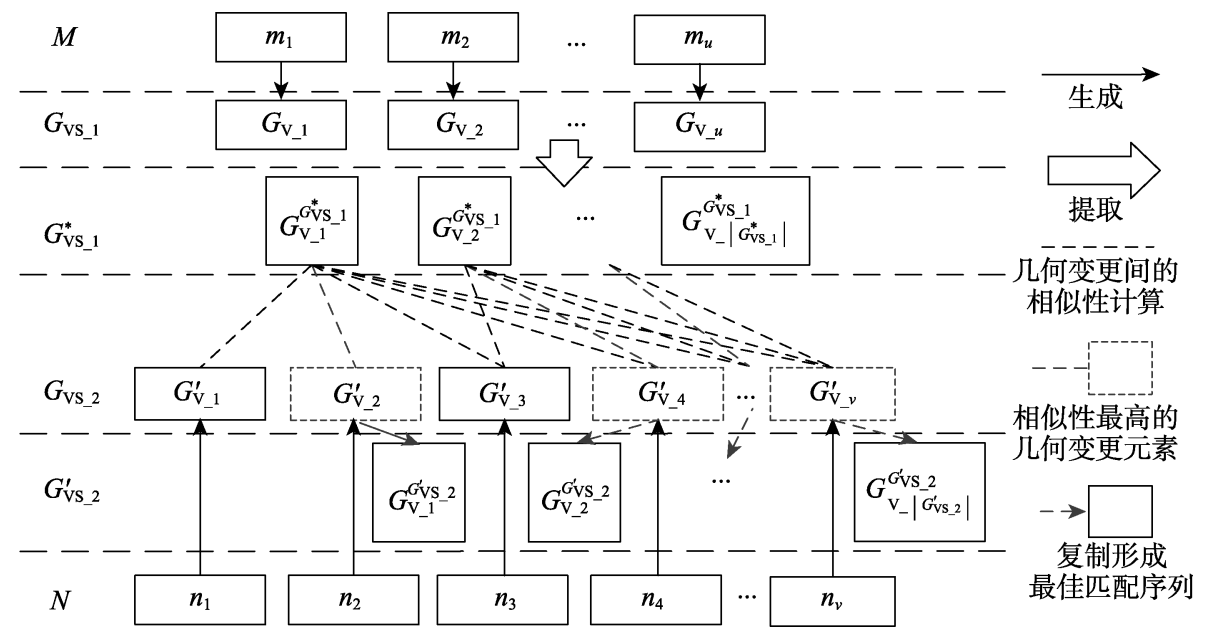

图 4 最佳匹配序列的获取过程

计算 $G_{\mathrm{VS} 1}^{*}$ 和 $G_{\mathrm{VS} 2}^{\prime}$ 的相似性，考虑 $G_{\mathrm{VS} 2}^{\prime}$ 的序 列长度可能与 $G_{\mathrm{VS} \_1}^{*}$ 的长度不一致的情况, 并进行 修订，具体计算公式为

$$
\begin{aligned}
& S_{\text {im }}^{\prime}\left(G_{\mathrm{VS}_{-} 1}, G_{\mathrm{VS}_{-} 2}\right)=
\end{aligned}
$$

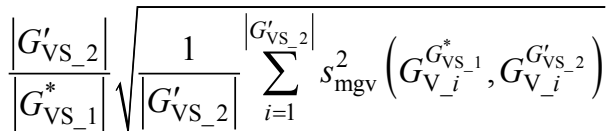

其中, $\left|G_{\mathrm{VS}_{-} 2}^{\prime}\right|$ 为 $G_{\mathrm{VS}_{-} 2}^{\prime}$ 中包含的几何变更数量; $\left|G_{\mathrm{VS} \_1}^{*}\right|$ 为 $G_{\mathrm{VS} \_1}^{*}$ 中包含的几何变更数量; $G_{\mathrm{V}_{-} i}^{G_{\mathrm{VS} 1}^{*}}$ 为 $G_{\mathrm{VS} 1}^{*}$ 中的第 $i$ 个几何变更; $G_{\mathrm{V}_{-}-2}^{G^{\prime}{ }_{2}}$ 为 $G_{\mathrm{VS} \_}^{\prime}$ 中的第 $i$ 个几何变更.

\section{3 多维度融合的工艺过程相似性计算}

对于 2 条加工方法序列 $M$ 和 $N$, 如果匹配构造 出它们对应的几何演变序列 $G_{\mathrm{VS}_{-} 1}$ 和 $G_{\mathrm{VS} \_2}$, 则可 以计算出它们对应的加工方法序列相似性 $S_{\mathrm{im}}(M, N)$ 和几何演变过程的相似性 $S_{\mathrm{im}}^{\prime}\left(G_{\mathrm{VS} \_1}\right.$, $G_{\mathrm{VS} \_2}$ ). 在此基础上将二者进行融合, 可以实现 对工件机加工艺过程相似性的多角度和全方位评 价，具体计算公式为

$$
S_{\mathrm{IM}}(M, N)=\omega S_{\text {im }}(M, N)+\omega^{\prime} S_{\text {im }}^{\prime}\left(G_{\mathrm{VS}_{-} 1}, G_{\mathrm{VS}_{-} 2}\right)
$$

其中, $S_{\mathrm{IM}}(M, N)$ 为 $M$ 和 $N$ 所对应的机加工艺过程 的多维相似性度量结果; $\omega$ 和 $\omega^{\prime}$ 分别为加工方法
序列相似性和几何演变过程相似性在多维融合计 算中的权重系数. 根据工艺人员的检索侧重, $\omega$ 和 $\omega^{\prime}$ 可以灵活赋值. 但从几何演变角度评价工艺 相似性是对传统工序序列方法的一种有效补充, 目的是使相似性的评判条件更为丰富, 增强检索 精度. 所以在具体赋值时, 一个启发性原则是 $\omega^{\prime}$ 的取值不宜高于 $\omega$. 经过多次篮选发现, 当 $\omega$ 的 权重系数为 0.6 且 $\omega^{\prime}$ 的权重系数为 0.4 时, 检索出 的相似案例与工艺人员认知的最为相似案例一致, 所以本文取 $\omega=0.6, \omega^{\prime}=0.4$.

\section{4 实例验证}

\section{1 实例 I}

以图 5 图 7 所示的零件为例进行验证. 其中, 图 5 和图 6 所示均为轴类零件, 图 7 所示为盘盖类 零件. 计算图 5 所示零件的工艺过程, 并与图 6 和 图 7 所示零件的工艺过程间的多维相似性度量值 进行比较分析.

\subsection{1 加工方法序列的相似性计算}

对图 5 图 7 所示零件的工艺过程进行分析, 提取其主要机械加工工序如表 2 所示.

根据表 2, 提取 $M$ 和 $N$ 的最长公共子序列 $S$ (粗 车 $\rightarrow$ 半精车 $\rightarrow$ 精车 $\rightarrow$ 粗铣/铁削), $M$ 和 $N^{\prime}$ 的最长公 共子序列 $S^{\prime}$ (粗车 $\rightarrow$ 半精车 $\rightarrow$ 粗铣/铣削). 然后利 用式(2) 式(6), 可以得到加工序列相似性度量结 果, 如表 3 所示. 

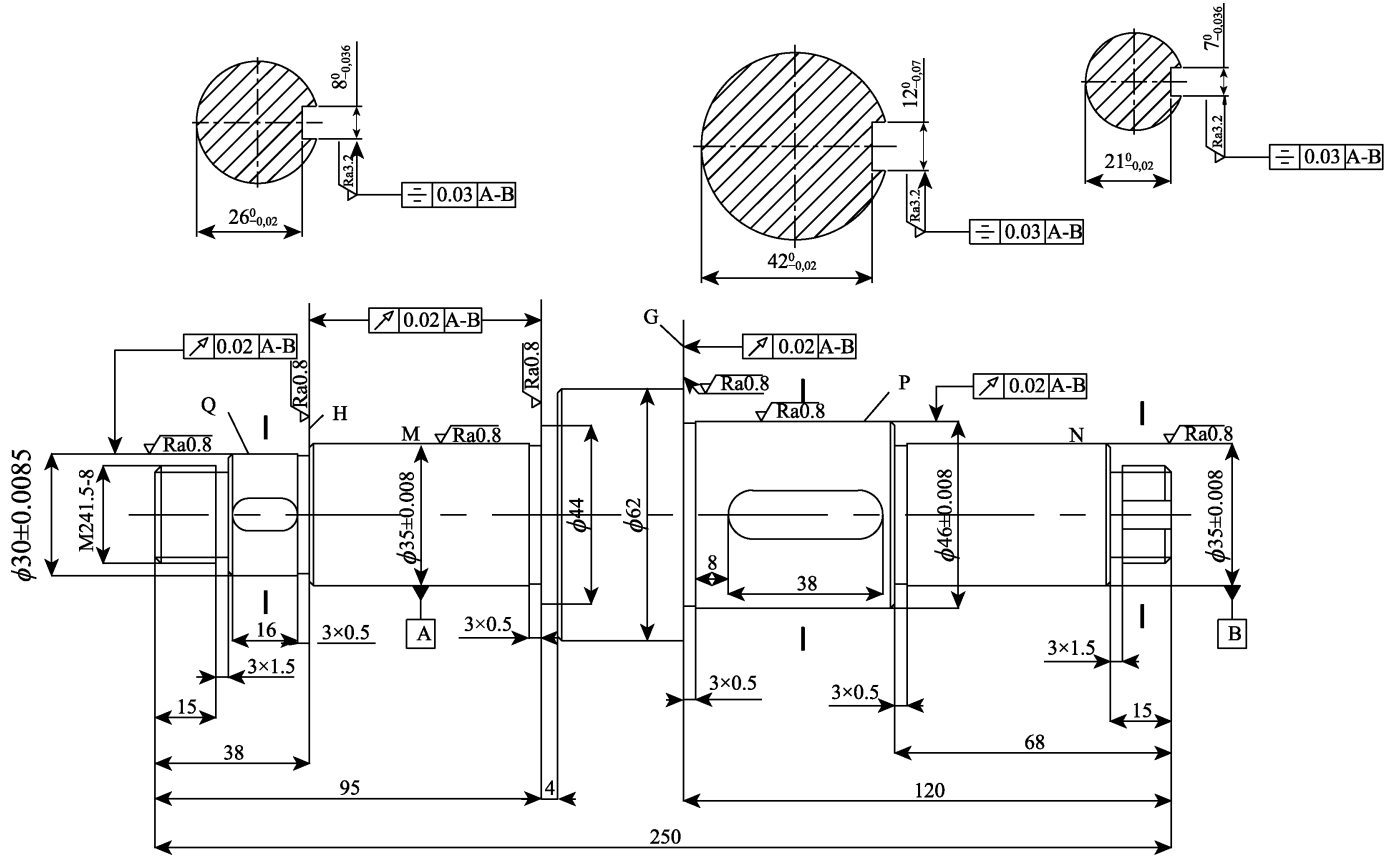

图 5 某传动轴零件的 $\mathrm{CAD}$ 设计图

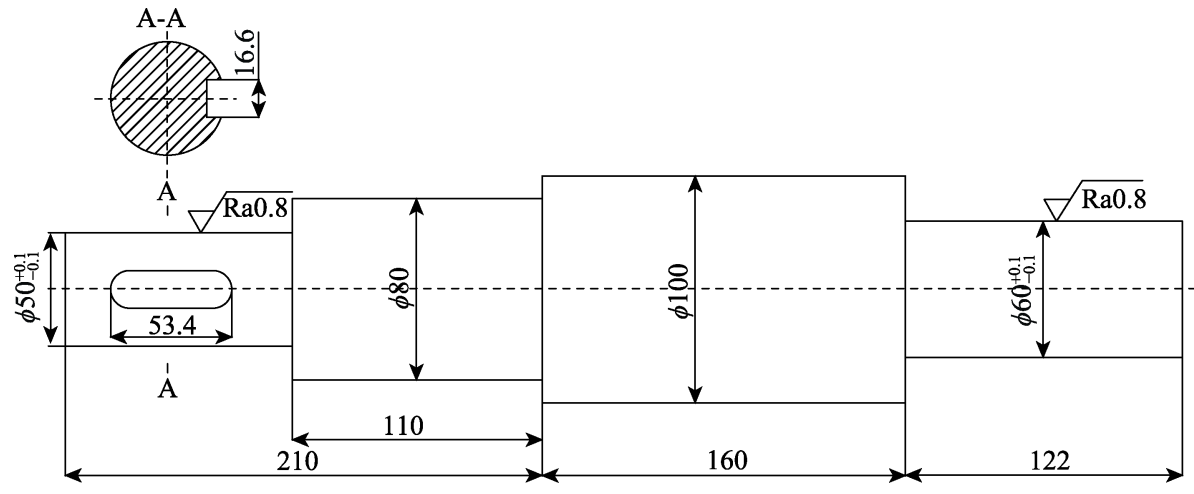

图 6 某阶梯轴零件的 $\mathrm{CAD}$ 设计图

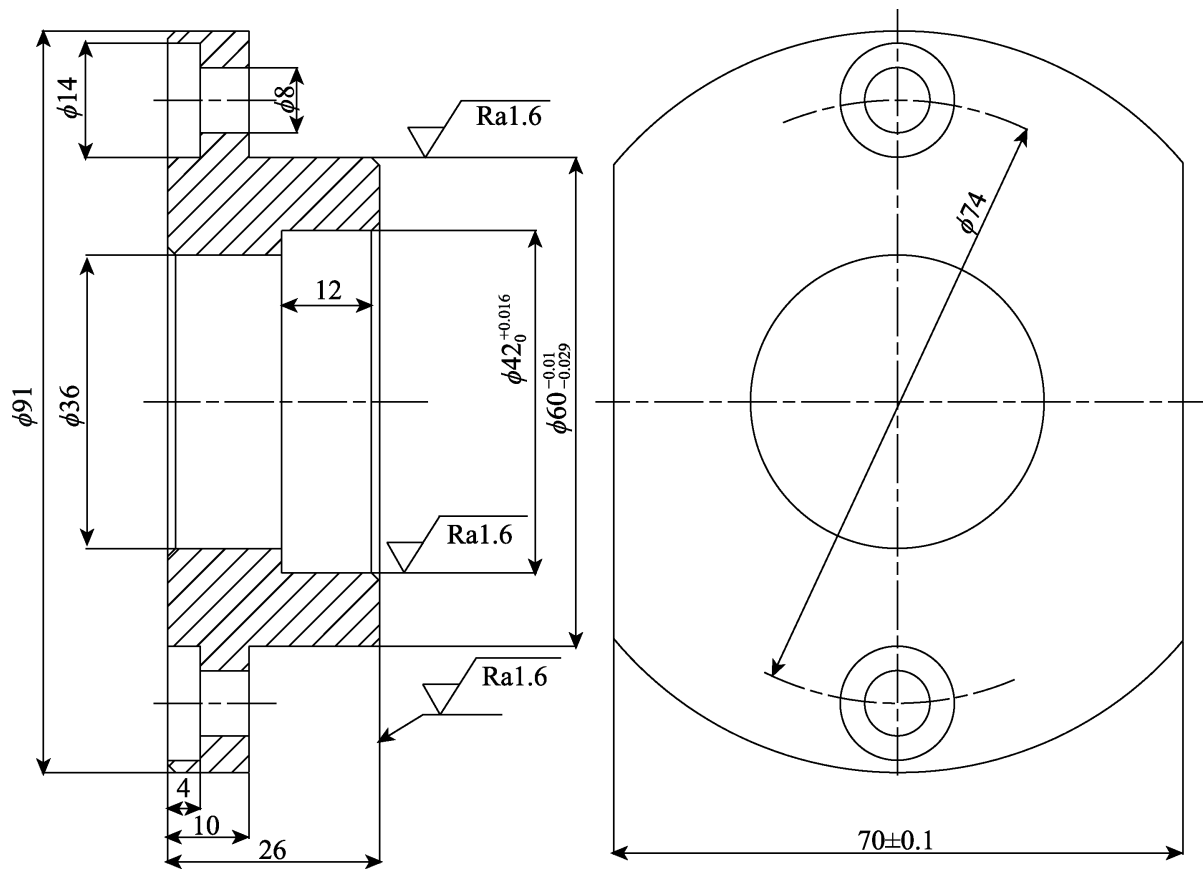

图 7 某盘盖零件的 CAD 设计图 
表 2 零件实例的加工方法序列

\begin{tabular}{ccl}
\hline 零件实例 & 加工方法序列代号 & \multicolumn{1}{c}{ 加工方法序列 } \\
\hline 图 5 & $M$ & $\begin{array}{l}\text { 粗车 } \rightarrow \text { 半精车 } \rightarrow \text { 精车 } \rightarrow \text { 粗铣 } \\
\rightarrow \text { 半精铣 } \rightarrow \text { 精铣 } \rightarrow \text { 磨 }\end{array}$ \\
图 6 & $N$ & $\begin{array}{l}\text { 粗车 } \rightarrow \text { 半精车 } \rightarrow \text { 精车 } \rightarrow \text { 铁削 } \\
\text { 图 } 7\end{array}$ \\
\hline
\end{tabular}

表 3 加工方法序列的相似性计算结果

\begin{tabular}{cc}
\hline$X_{\mathrm{S}}$ & $S_{\mathrm{im}}\left(M, X_{\mathrm{S}}\right)$ \\
\hline$N$ & 0.5714 \\
$N^{\prime}$ & 0.4268 \\
\hline
\end{tabular}

\subsection{2 几何演变序列的相似性计算}

根据加工方法序列与几何演变序列间的对应 关系, 可以对表 2 所示 3 个加工方法序列的对应几 何演变序列进行构造. 其中, $M$ 对应的几何演变序 列为 $G_{\mathrm{VS} 1}, N$ 对应的几何演变序列为 $G_{\mathrm{VS} 22}, N^{\prime}$ 对应的几何演变序列为 $G_{\mathrm{VS} 3}$.

进一步, 从 $G_{\mathrm{VS} \_1}$ 中可以提取得到 $G_{\mathrm{VS} \_1}^{*}$, 如 图 8 所示, 并以 $G_{\mathrm{VS} 11}^{*}$ 为比较基础, 从 $G_{\mathrm{VS} 22}$ 和 $G_{\mathrm{VS} 3}$ 中获取如图 9 所示最佳匹配序列 $G_{\mathrm{VS} 2}^{\prime}$ 和图 10 所示 $G_{\mathrm{VS} 3}^{\prime}$.

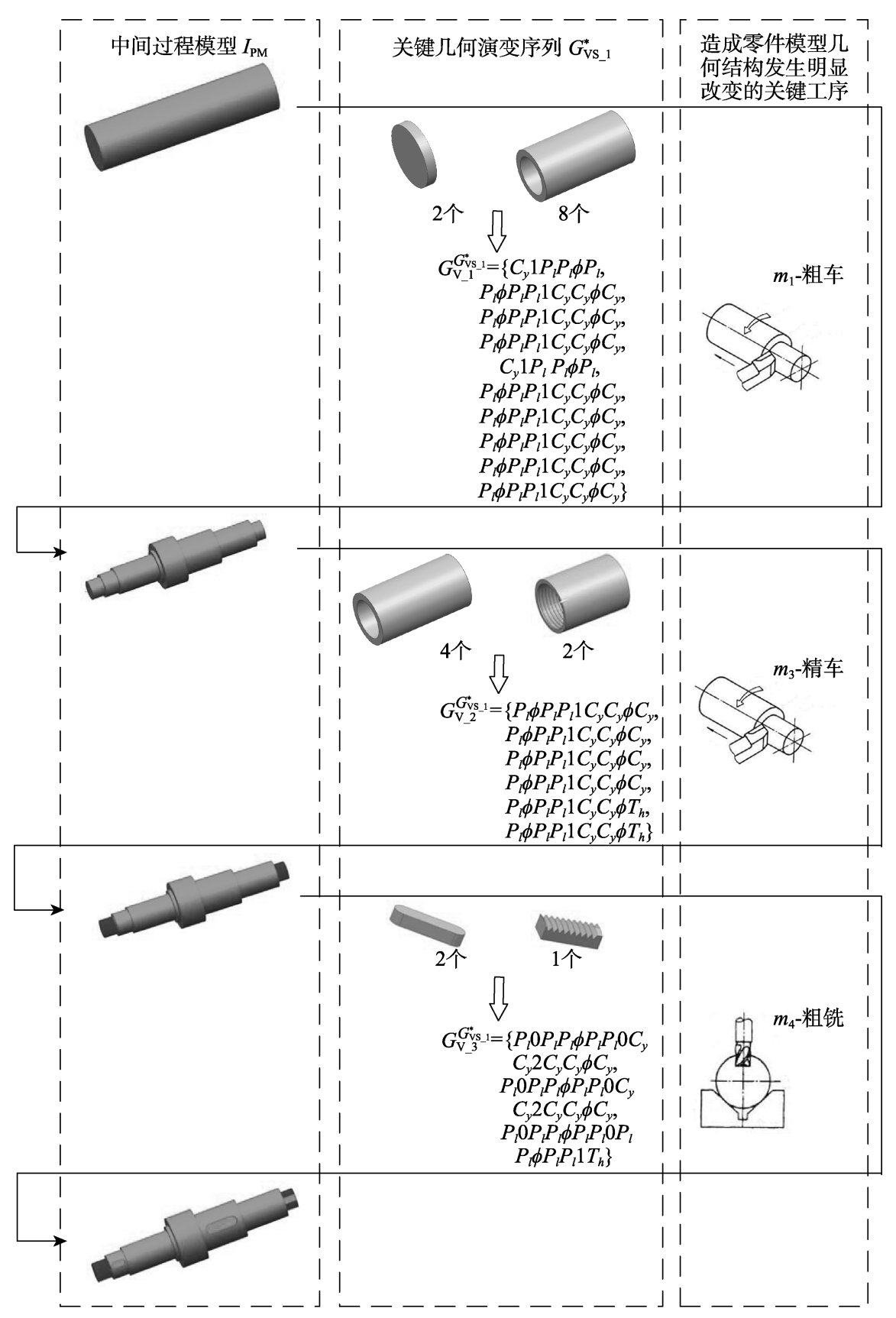

图 8 匹配 $M$ 的关键几何演变序列 $G_{\mathrm{VS} \_1}^{*}$ 


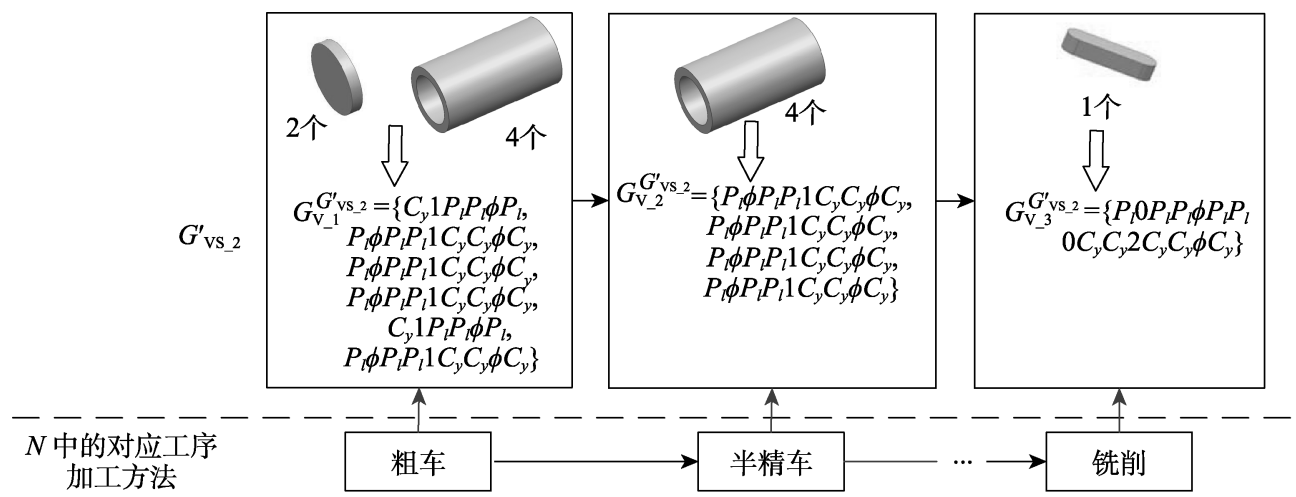

图 9 最佳匹配序列 $G_{\mathrm{VS} \text { _2 }}^{\prime}$

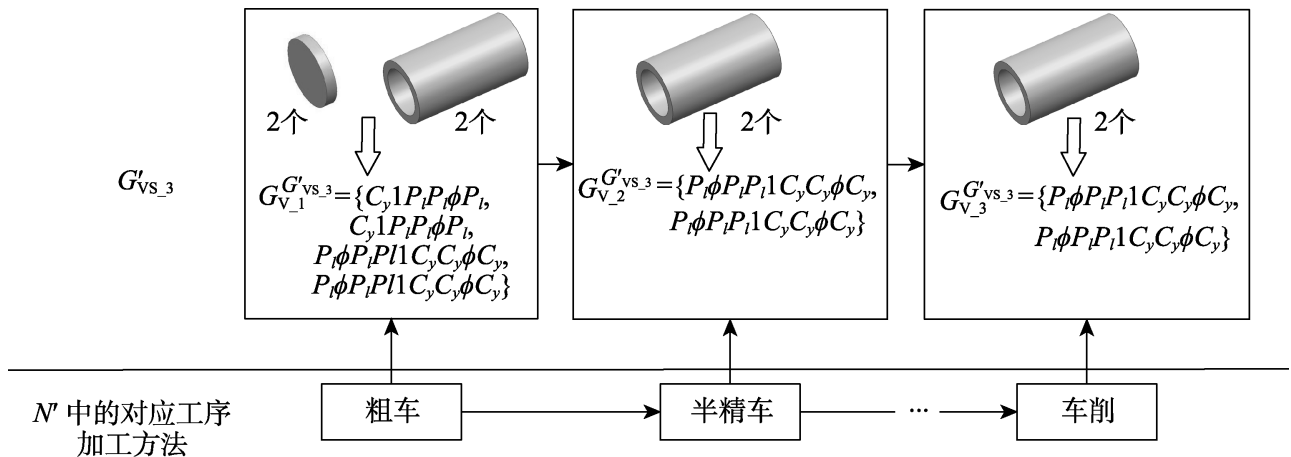

图 10 最佳匹配序列 $G_{\mathrm{VS} \_3}^{\prime}$

根据式(10)分别计算 $G_{\mathrm{VS} \_1}$ 与 $G_{\mathrm{VS} \_2}, G_{\mathrm{VS} \_3}$ 间 的相似性, 即从伴随机加过程的几何演变角度出 发，对因采用加工方法序列 $M, N$ 和 $N^{\prime}$ 所导致的工 件几何结构的演变过程进行相似性评判, 计算结 果如表 4 所示.

表 4 几何演变序列的相似性计算结果

\begin{tabular}{cc}
\hline$i$ & $S_{\mathrm{im}^{\prime}}^{\prime}\left(G_{\mathrm{VS}_{-} 1}, G_{\mathrm{VS}_{-}}\right)$ \\
\hline 2 & 0.5524 \\
3 & 0.3323 \\
\hline
\end{tabular}

4.1.3 多维融合的工艺过程相似性度量结果及分析 将表 3 和表 4 中相似性值代人式(11), 得到工 艺过程的多维相似性度量结果, 如表 5 所示.

表 5 工艺过程的多维度相似性度量结果

\begin{tabular}{cc}
\hline$X_{\mathrm{P}}$ & $S_{\mathrm{IM}}\left(M, X_{\mathrm{P}}\right)$ \\
\hline$N$ & 0.5638 \\
$N^{\prime}$ & 0.3890 \\
\hline
\end{tabular}

对比表 3 表 5 中数据可以发现, 多维相似性 计算值比单纯从加工方法序列角度计算得到的相 似性值有所降低. 这主要是因为本文采用了基于 符号熵的字符序列相似性计算方法，不仅充分体 现待比较加工方法序列的最长公共子序列在相似
性计算中的作用，而且综合考虑了共有加工工序 在原序列中的出现频次和分布密度等信息, 避免 了单纯依靠公共子序列计算相似性带来的未考虑 原序列整体结构信息的问题，即兼顾了原序列整 体结构的相似性和公共子序列的局部相似性. 与 传统依靠最长公共子序列等方式进行度量的结果 相比, 从加工方法序列角度进行相似性度量的计 算值偏低，而融人了机加工艺过程的几何演变序 列相似性计算结果后，因为同种工序加工方法可 能造成工件模型的几何结构改变有所差异, 所以 会造成从多维角度得到的相似性计算值进一步降 低. 这些都保证了在进行工艺相似性搜索时可以 从多个维度对待检工艺过程之间的差异性进行精 准刻画和全面体现, 使检测出相似性较高的工艺 案例真正贴合检索需求，避免了相似性搜索时的 “过检”和“误判”问题. 同时，本文还发现，无论是 从加工方法序列角度、工艺过程的几何演变角度还 是从多维相似性值的融合角度, 得到的 $M$ 与 $N$ 的 相似性计算值均比 $M$ 与 $N^{\prime}$ 的高, 这也与“同类零 件的工艺过程具有较高相似性” 的经验认知相一 致，说明了本文方法的有效性.

\section{2 实例 II}

为了更充分证明本文方法的有效性，随机抽 选 10 个零件进行机加工艺分析. 
计算这 10 个实例彼此间的多维工艺相似性值, 如图 11 所示. 由图 11 可知, 排除实例自身与自身 的相似性完全一致的情况，基本同类零件的工艺 过程相似性明显大于非同类零件的工艺相似性. 例如, 1 号零件与 2 号零件同属轴类零件, 彼此的 工艺过程相似性最大； 3 号零件与 4 号零件同属套

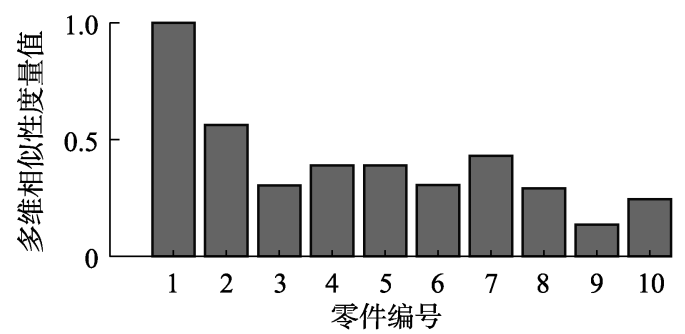

a. 1 号零件与其他零件的相似性度量值

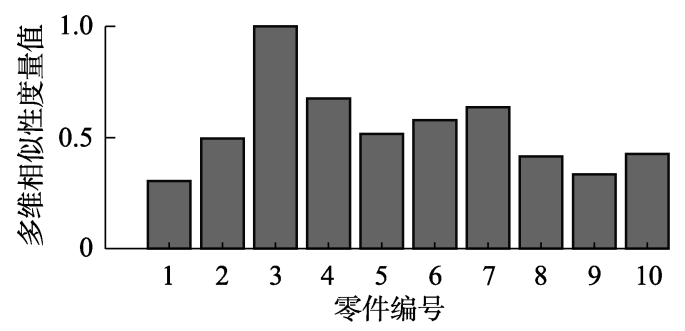

c. 3 号零件与其他零件的相似性度量值

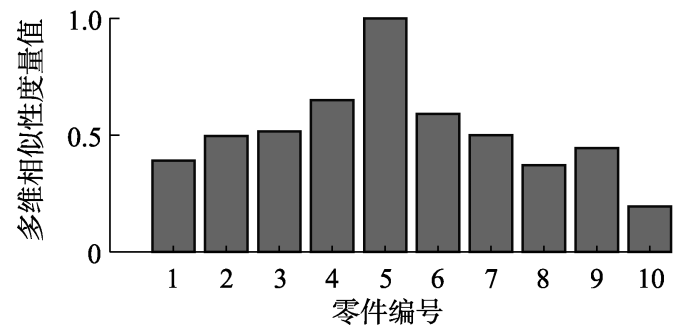

e. 5 号零件与其他零件的相似性度量值

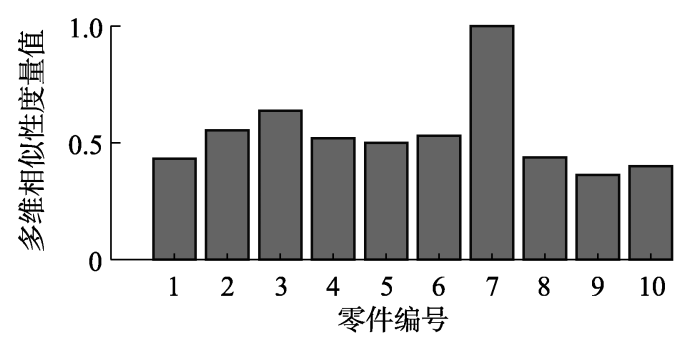

g. 7 号零件与其他零件的相似性度量值

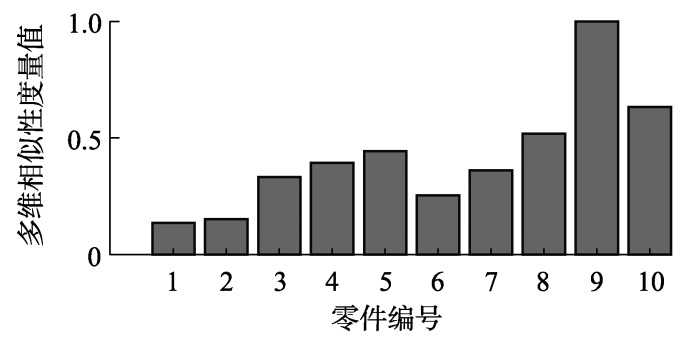

i. 9 号零件与其他零件的相似性度量值
类零件, 彼此的工艺过程相似性最大; 9 号零件与 10 号零件同属箱体类零件, 彼此的工艺过程相似 性最大. 这与企业的实际制造工艺过程和经验认 知相一致, 证明了本文方法的有效性.

与实例 I 同理获取加工方法序列和几何演变 序列，如表 6 所示.

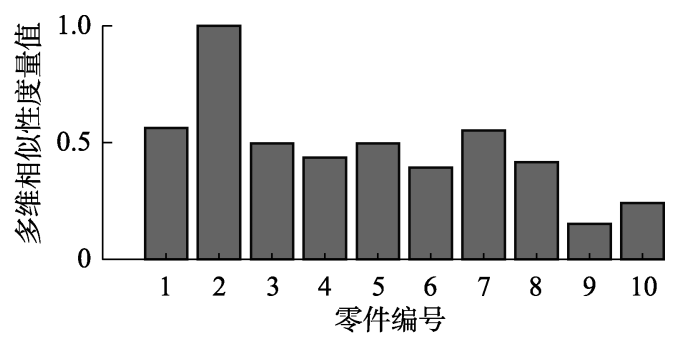

b. 2 号零件与其他零件的相似性度量值

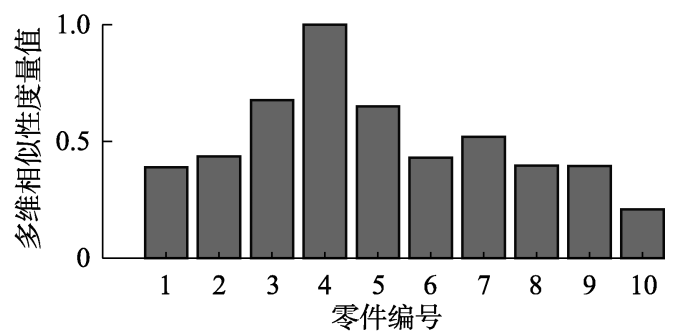

d. 4 号零件与其他零件的相似性度量值

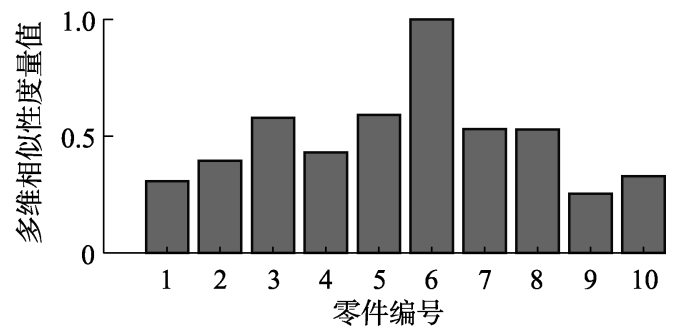

f. 6 号零件与其他零件的相似性度量值

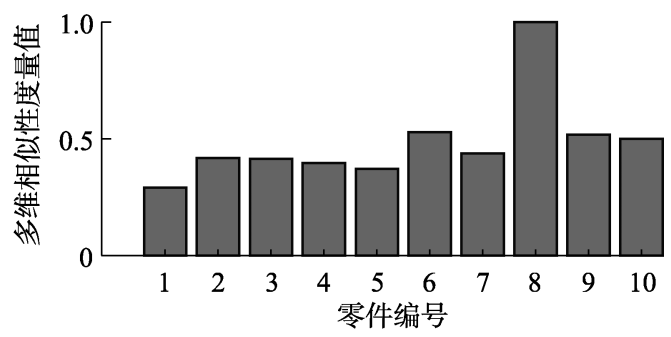

h. 8 号零件与其他零件的相似性度量值

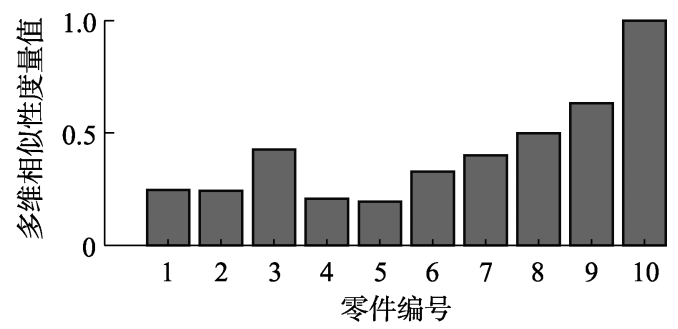

j. 10 号零件与其他零件的相似性度量值

图 11 实例 II 的工艺过程多维相似性计算结果 
表 6 实例 II 提取到的工艺实例

\begin{tabular}{|c|c|c|c|}
\hline 编号 & 零件名称 & 加工方法序列 & 关键几何演变序列 \\
\hline 1 & 传动轴 & $\begin{array}{l}\text { 粗车 } \rightarrow \text { 半精车 } \rightarrow \text { 精车 } \rightarrow \text { 粗铣 } \rightarrow \text { 半 } \\
\text { 精铣 } \rightarrow \text { 精铣 } \rightarrow \text { 磨 }\end{array}$ & $\begin{array}{l}\left\{2 \text { 个 } C_{y} 1 P_{l} P_{l} \phi P_{l}, 8 \text { 个 } P_{l} \phi P_{l} P_{l} 1 C_{y} C_{y} \phi C_{y}\right\} \rightarrow\left\{4 \text { 个 } P_{l} \phi P_{l} P_{l} 1 C_{y} C_{y} \phi C_{y}, 2 \text { 个 } P_{l} \phi P_{l} P_{l} 1 C_{y} C_{y} \phi T_{h}\right\} \\
\rightarrow\left\{2 \text { 个 } P_{l} 0 P_{l} P_{l} \phi P_{l} P_{l} 0 C_{y} C_{y} 2 C_{y} C_{y} \phi C_{y}, 1 \text { 个 } P_{l} 0 P_{l} P_{l} \phi P_{l} P_{l} 0 P_{l} P_{l} \phi P_{l} P_{l} 1 T_{h}\right\}\end{array}$ \\
\hline 2 & 阶梯轴 & 粗车 $\rightarrow$ 半精车 $\rightarrow$ 精车 $\rightarrow$ 铣削 & $\left\{2\right.$ 个 $C_{y} 1 P_{l} P_{l} \phi P_{l}, 4$ 个 $\left.P_{l} \phi P_{l} P_{l} 1 C_{y} C_{y} \phi C_{y}\right\} \rightarrow\left\{1\right.$ 个 $\left.P_{l} 0 P_{l} P_{l} \phi P_{l} P_{l} 0 C_{y} C_{y} 2 C_{y} C_{y} \phi C_{y}\right\}$ \\
\hline 3 & 气缸套 & 铸造 $\rightarrow$ 粗车 $\rightarrow$ 半精车 $\rightarrow$ 镗削 & $\left\{2\right.$ 个 $C_{y} 1 P_{l} P_{l} \phi P_{l}, 2$ 个 $\left.P_{l} \phi P_{l} P_{l} 1 C_{y} C_{y} \phi C_{y}\right\} \rightarrow\left\{1\right.$ 个 $\left.C_{y} 1 P_{l} P_{l} \phi P_{l}\right\}$ \\
\hline 4 & 导套 & 粗车 $\rightarrow$ 半精车 $\rightarrow$ 钻 $\rightarrow$ 镗削 $\rightarrow$ 磨 & $\left\{2\right.$ 个 $C_{y} 1 P_{l} P_{l} \phi P_{l}, 2$ 个 $\left.P_{l} \phi P_{l} P_{l} 1 C_{y} C_{y} \phi C_{y}\right\} \rightarrow\left\{2\right.$ 个 $\left.C_{y} 1 P_{l} P_{l} \phi P_{l}\right\} \rightarrow\left\{2\right.$ 个 $\left.P_{l} \phi P_{l} P_{l} 1 C_{y} C_{y} \phi C_{y}\right\}$ \\
\hline 5 & 轴承盖 & $\begin{array}{l}\text { 粗车 } \rightarrow \text { 半精车 } \rightarrow \text { 钻 } \rightarrow \text { 车削 (内 } \\
\text { 圆) } \rightarrow \text { 铣削 } \rightarrow \text { 钻 }\end{array}$ & $\begin{array}{l}\left\{2 \text { 个 } C_{y} 1 P_{l} P_{l} \phi P_{l}, 2 \text { 个 } P_{l} \phi P_{l} P_{l} 1 C_{y} C_{y} \phi C_{y}\right\} \rightarrow\left\{2 \text { 个 } C_{y} 1 P_{l} P_{l} \phi P\right\} \rightarrow\left\{2 \text { 个 } P_{l} \phi P_{l} P_{l} 1 C_{y} C_{y} \phi C_{y}\right\} \rightarrow \\
\left\{2 \text { 个 } P_{l} 0 C_{y} C_{y} 1 P_{l} P_{l} \phi P_{l}\right\} \rightarrow\left\{4 \text { 个 } C_{y} 1 P_{l} P_{l} \phi P_{l}\right\}\end{array}$ \\
\hline 6 & 法兰盘 & $\begin{array}{l}\text { 铸造 } \rightarrow \text { 粗车 } \rightarrow \text { 半精车 } \rightarrow \text { 精车 } \rightarrow \text { 钻 } \\
\rightarrow \text { 粗车 }(\text { 内圆 }) \rightarrow \text { 精车 }(\text { 内圆 }) \rightarrow \text { 钻 }\end{array}$ & $\begin{array}{l}\left\{2 \text { 个 } C_{y} 1 P_{l} P_{l} \phi P_{l}, 1 \text { 个 } P_{l} \phi P_{l} P_{l} 1 C_{y} C_{y} \phi C_{y}\right\} \rightarrow\left\{1 \text { 个 } C_{y} 1 P_{l} P_{l} \phi P\right\} \rightarrow\left\{1 \text { 个 } P_{l} \phi P_{l} P_{l} 1 C_{y} C_{y} \phi C_{y}\right\} \\
\rightarrow\left\{6 \text { 个 } C_{y} 1 P_{l} P_{l} \phi P_{l}\right\}\end{array}$ \\
\hline 7 & 阀盖 & $\begin{array}{l}\text { 铸造 } \rightarrow \text { 粗车 } \rightarrow \text { 半精车 } \rightarrow \text { 精车 } \rightarrow \text { 镗 } \\
\text { 削(中心孔) } \rightarrow \text { 钻 }\end{array}$ & $\left\{2\right.$ 个 $C_{y} 1 P_{l} P_{l} \phi P_{l}, 3$ 个 $\left.P_{l} \phi P_{l} P_{l} 1 C_{y} C_{y} \phi C_{y}\right\} \rightarrow\left\{1\right.$ 个 $\left.C_{y} 1 P_{l} P_{l} \phi P_{l}\right\} \rightarrow\left\{4\right.$ 个 $\left.C_{y} 1 P_{l} P_{l} \phi P_{l}\right\}$ \\
\hline 8 & 闪盖 & $\begin{array}{l}\text { 铸造 } \rightarrow \text { 粗车 } \rightarrow \text { 半精车 } \rightarrow \text { 铣削 } \rightarrow \\
\text { 钻(扩) }\end{array}$ & $\left\{1\right.$ 个 $\left.P_{l} \phi P_{l} P_{l} 1 C_{y} C_{y} \phi C_{y}\right\} \rightarrow\left\{1\right.$ 个 $\left.P_{l} \phi P_{l} P_{l} 1 C_{y} C_{y} \phi C_{y}\right\} \rightarrow\left\{8\right.$ 个 $\left.C_{y} 1 P_{l} P_{l} \phi P_{l}\right\}$ \\
\hline 9 & 角形轴承箱 & $\begin{array}{l}\text { 铸造 } \rightarrow \text { 铣削 } \rightarrow \text { 铣削 } \rightarrow \text { 粗镗 } \rightarrow \text { 精 } \\
\text { 镗 } \rightarrow \text { 铣削 } \rightarrow \text { 钻 } \rightarrow \text { 铣削 } \rightarrow \text { 钻 }\end{array}$ & $\begin{array}{l}\left\{2 \text { 个 } P_{l} \phi P_{l} P_{l} 0 P_{l} P_{l} \phi P_{l} P_{l} 0 P_{l} P_{l} \phi P_{l}\right\} \rightarrow\left\{2 \text { 个 } P_{l} \phi P_{l} P_{l} 0 P_{l} P_{l} \phi P_{l} P_{l} 0 P_{l} P_{l} \phi P_{l}\right\} \rightarrow\left\{1 \text { 个 } P_{l} \phi P_{l} P_{l} 1 C_{y}\right. \\
\left.C_{y} \phi C_{y}\right\} \rightarrow\left\{2 \text { 个 } P_{l} \phi P_{l} P_{l} \quad 1 C_{y} C_{y} \phi C_{y}\right\} \rightarrow\left\{6 \text { 个 } C_{y} 1 P_{l} P_{l} \phi P_{l}\right\} \rightarrow\left\{2 \text { 个 } P_{l} \phi P_{l} P_{l} 0 P_{l} P_{l} \phi P_{l} P_{l} 0 P_{l} P_{l}\right. \\
\left.\phi P_{l}\right\} \rightarrow\left\{2 \text { 个 } C_{y} 1 P_{l} P_{l} \phi P_{l}\right\}\end{array}$ \\
\hline 10 & 阀体 & $\begin{array}{l}\text { 铸造 } \rightarrow \text { 铣削 } \rightarrow \text { 铁削 } \rightarrow \text { 铣削 } \rightarrow \text { 粗 } \\
\text { 镗 } \rightarrow \text { 精镗 } \rightarrow \text { 粗镗 } \rightarrow \text { 钻 }(\text { 攻螺纹 }) \rightarrow \\
\text { 钻(攻螺纹) }\end{array}$ & $\begin{array}{l}\left\{2 \text { 个 } P_{l} \phi P_{l} P_{l} 1 C_{y} C_{y} \phi C_{y}\right\} \rightarrow\left\{1 \text { 个 } P_{l} \phi P_{l} P_{l} 1 C_{y} C_{y} \phi C_{y}\right\} \rightarrow\left\{1 \text { 个 } P_{l} \phi P_{l} P_{l} 1 C_{y} C_{y} \phi C_{y}\right\} \rightarrow\{6 \text { 个 } \\
\left.P_{l} \phi P_{l} P_{l} 1 C_{y} C_{y} \phi C_{y}\right\} \rightarrow\left\{1 \text { 个 } P_{l} \phi P_{l} P_{l} 1 C_{y} C_{y} \phi C_{y}\right\} \rightarrow\left\{1 \text { 个 } C_{y} 1 P_{l} P_{l} \phi P_{l}\right\} \rightarrow\left\{8 \text { 个 } C_{y} 1 P_{l} P_{l} \phi P_{l}\right\}\end{array}$ \\
\hline
\end{tabular}

\section{5 结 语}

本文从工序加工方法序列的相似性及其对应 的几何演变过程的相似性角度出发, 构建了机加 工艺过程的多维度相似性度量方法，在一定程度 上促进了相似工艺案例的检索质效提升，同时对 挖掘同类案例背后隐藏的共性知识和规律以支持 制造过程中的智能化重用具有借鉴意义.

当然, 制造加工过程的组织安排在强调智能 性的同时, 也要综合权衡设计人员对工艺过程组 织安排的潜在倾向性，虽然本文方法实现了对工 艺过程的多维度相似性计算, 但后续还需进一步 研究更贴近工艺人员主观设计意图的检索新模式, 在确保检索效率的前提下, 不断融人新的检索维 度，不断提升工艺搜索方式的灵活性与检索结果 的可靠度.

\section{参考文献(References):}

[1] Liu S N, Zhang Z M, Tian X T. A typical process route discovery method based on clustering analysis[J]. The International Journal of Advanced Manufacturing Technology, 2007, 35: 186-194

[2] Zhang Hui, Qiu Lemiao, Zhang Shuyou, et al. Typical product process route extraction method based on intelligent clustering analysis[J]. Computer Integrated Manufacturing Systems, 2013, 19(3): 490-498(in Chinese)

(张辉，表乐录，张树有，等. 基于智能聚类分析的产品典型
工艺路线提取方法 [J]. 计算机集成制造系统，2013，19(3): 490-498)

[3] Zhou Danchen. Typical process route discovery method based on granular computing and sequence alignment[J]. Computer Integrated Manufacturing Systems, 2015, 21(3): 694-701(in Chinese)

(周丹晨. 基于粒计算和序列比对的典型工艺路线发现方法 [J]. 计算机集成制造系统, 2015, 21(3): 694-701)

[4] Fan Wuyang. Research on machining process knowledge mining and reuse method of spacecraft part[D]. Harbin: Harbin Institute of Technology, 2019(in Chinese)

(范午阳. 航天器零件机加工艺知识挖掘与重用方法研究 [D].哈尔滨: 哈尔滨工业大学, 2019)

[5] Cao Yong. Research on process knowledge discovering and reusing based on data mining[D]. Jinan: Shandong University, 2019(in Chinese)

(曹勇. 基于数据挖掘的工艺知识发现与重用研究 [D]. 济南: 山东大学, 2019)

[6] El-Mehalawi M, Miller R A. A database system of mechanical components based on geometric and topological similarity. Part I: representation[J]. Computer-Aided Design, 2003, 35(1): 83-94

[7] El-Mehalawi M, Miller R A. A database system of mechanical components based on geometric and topological similarity. Part II: indexing, retrieval, matching, and similarity assessment[J]. Computer-Aided Design, 2003, 35(1): 95-105

[8] Cuillière J C, François V, Souaissa K, et al. Automatic comparison and remeshing applied to CAD model modification[J]. Computer-Aided Design, 2011, 43(12): 1545-1560

[9] Liu Jihong, Hou Yongzhu. MBD model parametric method based on hybrid-attributed adjacency graph[J]. Journal of Computer-Aided Design \& Computer Graphics, 2018, 30(7): 1329-1334(in Chinese) 
(刘继红, 侯永柱. 基于混合属性邻接图的 MBD 模型参数化 方法 [J]. 计算机辅助设计与图形学学报, 2018, 30(7): 1329-1334)

[10] Wang Fei, Zhang Shusheng, Bai Xiaoliang, et al. Local matching of 3D CAD models based on subgraph isomorphism[J]. Journal of Computer-Aided Design \& Computer Graphics, 2008, 20(8): 1078-1084(in Chinese)

(王飞, 张树生, 白晓亮, 等. 基于子图同构的三维 CAD 模 型局部匹配[J]. 计算机辅助设计与图形学学报, 2008, 20(8): 1078-1084)

[11] Xie Fei, Guo Yu, Zhang Honglei, et al. Manufacturing feature recognition based on graph and subgraph isomorphism algorithm[J]. Journal of Nanjing University of Aeronautics and Astronautics, 2018, 50(3): 390-396(in Chinese)

(谢飞, 郭宇, 张红蕾, 等. 基于图和子图同构算法的制造特 征识别方法 [J]. 南京航空航天大学学报, 2018, 50(3): 390-396)

[12] Huo Xing, Tan Jieqing. 3D model retrieval based on feature vector[J]. Journal of Engineering Graphics, 2009, 30(3): 76-79(in Chinese)

(霍星，檀结庆. 利用特征向量的三维模型检索 [J]. 工程图 学学报, 2009, 30(3): 76-79)

[13] Zhou Yan, Zeng Fanzhi, Yang Yuewu. 3D model retrieval algorithm based on multi feature fusion[J]. Computer Science, 2016, 43(7): 303-309(in Chinese)

(周燕，曾凡智，杨跃武. 基于多特征融合的三维模型检索 算法 [J]. 计算机科学, 2016, 43(7): 303-309)

[14] Hu Shuhui, Yang Haicheng, Zhang Zhaojian, et al. Similarity measure of the geometry change sequence of three-dimensional process model[J]. Journal of Computer-Aided Design \& Computer Graphics, 2014, 26(7): 1176-1183(in Chinese) (胡淑慧, 杨海成, 张招建, 等. 三维工序模型几何演变序列 的相似性度量 $[\mathrm{J}]$. 计算机辅助设计与图形学学报, 2014, 26(7): 1176-1183)

[15] Li Chunlei, Mo Rong, Chang Zhiyong, et al. Generation method and applications of geometry variation sequence of intermediate process model[J]. Journal of Computer Aided Design \& Computer Graphics, 2017, 29(3): 565-574(in Chinese) (李春否, 莫蓉, 常智勇, 等. 零件工序模型几何演变序列生 成方法及应用 [J]. 计算机辅助设计与图形学学报, 2017, 29(3): 565-574)

[16] Chang Zhiyong, Tao Lizun, Li Jiajia, et al. The measure and search method of process knowledge element based on machining intent[J]. Journal of Mechanical Engineering, 2018, 54(3): 160-168(in Chinese)

(常智勇, 陶礼尊, 李佳佳, 等. 基于加工意图的机加工艺知 识重用方法研究[J]. 机械工程学报, 2018, 54(3): 160-168)
[17] Agrawal R, Faloutsos C, Swami A N. Efficient similarity search in sequence databases[C] //Proceedings of the 4th International Conference on Foundations of Data Organization and Algorithms. Heidelberg: Springer, 1993: 69-84

[18] Wu Donggen, Zhou Xiao'an. Similarity analysis of DNA sequences based on longest common subsequence[J]. Intelligent Computer and Applications, 2018, 8(6): 22-26(in Chinese) (吴东根, 周小安. 基于最长公共子序列的 DNA 序列相似性 分析[J]. 智能计算机与应用, 2018, 8(6): 22-26)

[19] Tian Fujun, Chen Xingyu, Cheng Wusi, et al. 3D machining process planning technology under model-based definition[J]. Computer Integrated Manufacturing Systems, 2014, 20(11): 2690-2696(in Chinese)

(田富君, 陈兴玉，程五四，等. MBD 环境下的三维机加工艺 设计技术 [J]. 计算机集成制造系统，2014，20(11)：26902696)

[20] Alemanni M, Destefanis F, Vezzetti E. Model-based definition design in the product lifecycle management scenario[J]. The International Journal of Advanced Manufacturing Technology, 2011, 52: 1-14

[21] Huang R, Zhang S S, Bai X L. Multi-level structuralized model-based definition model based on machining features for manufacturing reuse of mechanical parts[J]. The International Journal of Advanced Manufacturing Technology, 2014, 75: 1035-1048

[22] Huang Rui, Zhang Shusheng, Tao Jun, et al. An association method of precursory 3D process model and process drawing $[\mathrm{J}]$. Journal of Harbin Institute of Technology, 2012, 44(3): 102-106(in Chinese)

(黄瑞, 张树生, 陶俊, 等. 一种前驱三维工序模型与工序图 关联方法 [J]. 哈尔滨工业大学学报, 2012, 44(3): 102-106)

[23] Zhao Ming, Wang Xiyang. Rapid generation method of MBD process model based on volume decomposition[J]. Computer Integrated Manufacturing Systems, 2014, 20(8): 1843-1850(in Chinese)

(赵鸣, 王细洋. 基于体分解的 MBD 工序模型快速生成方法 [J]. 计算机集成制造系统, 2014, 20(8): 1843-1850)

[24] Chen Fei, Qiao Lihong. Approach to evolutionary construction of three dimensional operational model[J]. Aeronautical Manufacturing Technology, 2015(7): 82-85(in Chinese) (陈飞, 乔立红. 三维工序模型的演进式构建方法 [J]. 航空 制造技术, 2015(7): 82-85)

[25] Zhang Xin, Mo Rong, Shi Yuan, et al. String-based CAD models similarity assessment algorithm[J]. China Mechanical Engineering, 2009, 20(20): 2435-2440(in Chinese) (张欣, 莫蓉, 石源, 等. 基于字符串度量的 CAD 模型相似 性比较算法[J]. 中国机械工程, 2009, 20(20): 2435-2440) 